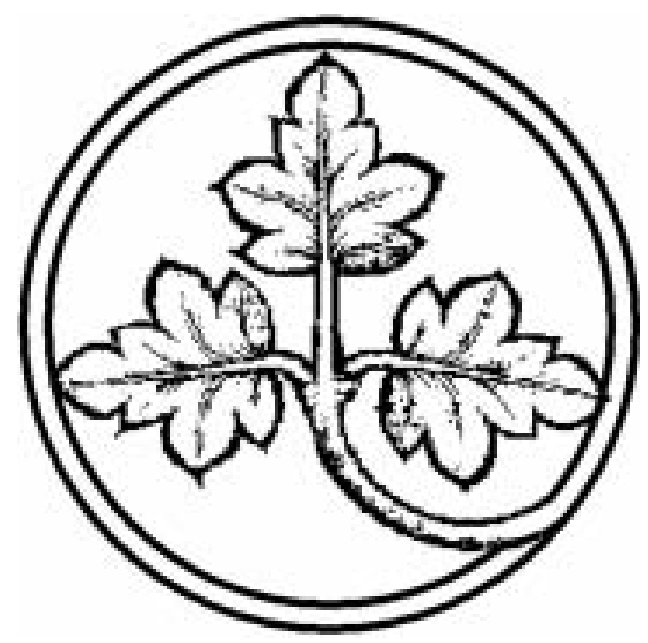

Gemeinschaftsgüter: Recht, Politik und Ökonomie

\author{
Preprints \\ aus der Max-Planck-Projektgruppe \\ Recht der Gemeinschaftsgüter \\ Bonn \\ $2003 / 2$
}

The Problems of Collective Action:

A New Approach

Von

Katharina Holzinger 


\title{
The Problems of Collective Action: A New Approach.
}

\author{
Katharina Holzinger
}

Bonn, Januar 2003

\begin{abstract}
The problem of collective action is usually identified with social dilemmas. A wider notion of the term collective action problem is introduced, as dilemmas are not the only problems to arise in collective action. The article first presents a typology of collective action problems based on matrix game analysis. Five types are distinguished: distribution, defection, co-ordination, disagreement, and instability problems. Second, the article discusses a number of proposals how to resolve these types of collective action problems, such as altruism, norms, focal points, correlated strategies, collective decision-making, external power, and sanctioning. Whereas the "political" solutions can be used to resolve all types of problems, the "motivational" solutions can only facilitate the resolution of some of the problems, and the "rational expectation" solutions can solve some types and help to solve others.
\end{abstract}

I gratefully acknowledge the comments on earlier versions of this paper given by Martin Beckenkamp, Max Planck Project Group on Common Goods, Bonn, Joachim Behnke, University of Bamberg, Christoph Engel and Adrienne Héritier, Max Planck Project Group on Common Goods, Alkuin Kölliker, Max Planck Project Group on Common Goods, Elinor Ostrom, Indiana University, Johannes Schmidt, University of Bamberg, Reinhard Zintl, University of Bamberg, and Michael Zürn, University of Bremen. 


\section{Introduction}

It was Mancur Olson's The Logic of Collective Action which made the notion of a collective action problem widely known in political and social science. Olson dealt with the provision of public goods by groups. He focussed on the non-excludability property of public goods, and thus, with the term collective action problem he mainly referred to the free rider problem in the organization of interests (Olson 1965: chapter I.B). Thereafter, collective action problems have mostly been identified with social dilemmas, that is, situations where rational individual actors are trapped to produce collectively sub-optimal results in terms of aggregated welfare. For example in Elster's "strong" definition, a collective action problem is the same as the prisoners' dilemma (Elster 1985). Taylor has shown that it is surely too restrictive to equate collective action problems with the prisoners' dilemma (Taylor 1987: 18). Taylor's own definition states "that a collective action problem exists where rational individual action can lead to a strictly Pareto-inferior outcome, that is, an outcome which is strictly less preferred by every individual than at least one other outcome" (Taylor 1987: 19; cf. also Liebrand and Messick 1996).

This definition, like most others, is only concerned with the welfare or Pareto-optimality aspects of collective action. Dilemmas between individual and collective rationality are, however, not the only problems to arise in collective action. This becomes obvious if one looks at game theory, or at different sub-disciplines in social science, like sociology, positive political theory, or empirical political research. Many other kinds of problem have been identified by social scientists, which arise in collective action or in the provision of public goods (see Scharpf 1997: chapter 4). To name a few: Pure conflict between the actors has been the subject of early game theory, dealing with zero sum games (von Neumann and Morgenstern 1943). Problems of coordination have been distinguished from problems of co-operation (Runge 1984; Ochs 1995; Rieck 1993: chapter 3; Braun 1999: chapter 3.3). Others have pointed to problems of finding agreement (Genschel and Plümper 1996) and to problems of inequality (Zürn 1992), which can arise in the strategic interaction of individuals trying to achieve some collective outcome. Finally, the instability of collective outcomes has been identified as a problem (for example Rieck 1993: chapter3). Inequality, instability, co-ordination failure, and sub-optimal outcomes are in general widely discussed in political and social science. The authors quoted here have in common that they link these problems to game theoretic models.

Against this background it seems to be justified to introduce a much wider notion of collective action problems than the ones cited above. In my definition the term "collective action" refers to the joint actions of a number of individuals which aim to achieve and distribute some gain through co-ordination or co-operation. The strategic constellations of actors can be such that they lead to difficulties in achieving the goals of collective action. All difficulties that arise in the pursuit of these goals and that are a consequence of strategic interaction will be called "collective action problems". This definition is not restricted to problems of inefficient outcomes, but includes, for example, problems of co-ordination, and inequality or instability of the collective 
outcome. Closest to this definition is the term "problematic situation", as used by Zürn (1992: 154ff.). ${ }^{1}$

This paper has two objectives: First and foremost, a systematic attempt will be made to classify the kinds of problems which can appear in collective action as a consequence of strategic interaction (section 2). Second, the paper will examine some motivational, rational, and political mechanisms which have been proposed as solutions to the respective collective action problems in philosophy and social science (section 3).

Basic types of strategic constellations in collective action can be represented by $2 \times 2$ matrix games - as has been done in the literature cited above. These models have the merit of demonstrating a given strategic structure very clearly. They are very good models of basic types of collective action problems and reveal a surprising amount of information on how these problems can be solved. In fact, matrix games are the most parsimonious means of analyzing collective action problems.

Therefore, section 2 starts with a classification of $2 \times 2$ matrix games which allows to distinguish games according to the types of collective action problems they pose. The classification is based on four game theoretic attributes that can easily be identified. From this classification of games a typology of five distinct problems of collective action can be derived. With its orientation towards collective action problems the typology is more relevant for political science than earlier taxonomies of matrix games concerned with stability or rationality of solutions, for example Rapoport et al. (1976). However, in its criteria the classification heavily relies on earlier taxonomies. Its own contribution is to synthesize this research from a political science perspective and to put it into a systematic framework.

The typology of collective action problems raises two general issues, a substantive and a methodological one. First, is there a correlation between the types of collective action problem and particular kinds of solution mechanisms which have been proposed in the literature? Second, are matrix games the right tool for representing collective action problems and are they a good basis for deriving solutions to them?

It is important, not to forget the restrictive assumptions, matrix games are based on. In the real world there are usually more than two actors and more than two strategies. Furthermore, in empirical situations players will often be able to communicate. Finally, in cases of repeated interaction and ongoing relationships among the actors, the single-shot game does not truly reflect the strategic situation: Many equilibria are possible if a game is played repeatedly. Therefore, after the types of basic collective action problems have been identified on the basis of strict matrix game theory in section 2, these assumptions will be discussed and systematically linked with the capacity to solve particular types of problem in section 3 .

1 It should be noted that this definition does not include all problems of collective decision-making. It excludes problems which are not the result of strategic behavior, for example, fundamental uncertainty about the consequences of alternative actions. Although such problems constitute another and very important problem of individual and also collective decision-making, they are not related to the strategic problems of collective action. 
There have not been many attempts in political science to relate social mechanisms and political institutions systematically to the various types of collective action problem. It is true that the implications for the solution of given strategic constellations have been discussed in game theory with regard to particular games and their properties, for example, coordination or defection problems or multiple equilibria games. Mixed strategies, correlated strategies, or the "mafia solution" of the prisoners' dilemma are examples for game theoretic proposals for solution mechanisms.

In philosophy, sociology and political science similar as well as different kinds of mechanisms have been proposed. For example, altruism and fairness norms are assumed to play a role in the resolution of collective action problems. In sociology, focal point solution, social conventions, and communication have been emphasized. In political science the solution of collective action problems by an external power, usually the state plays a dominant role. This includes not only mechanisms of collective decision-making, such as bargaining and voting, but also mechanisms for enforcing the decisions, such as monitoring and sanctioning.

The capacity of the various social and institutional mechanisms to resolve particular types of collective action problems will be tentatively analyzed. Although a number of propositions can be formulated on the basis of theory and previous research, a comprehensive analysis of all solution mechanisms and types of problems cannot be undertaken here. Thus, the results are preliminary. Future theory and empirical research has to fill in the gaps.

\section{The Problems of Collective Action}

\subsection{Taxonomies of Matrix Games}

In their theoretical history, matrix games have been classified according to different attributes. In the beginning, the distinction between zero-sum and non-zero-sum games became important (von Neumann and Morgenstern 1943). Later the number of Nash equilibria (one, two or more) served as a criterion (Nash 1951). Also, basic types of collective action problem played a role from the very beginning: Games were classified as prisoners' dilemmas, chicken or battle of the sexes games (cf. Kreps 1990: 37, Taylor 1987), as volunteers' dilemmas (Rapoport 1988), or as co-ordination and dis-coordination games (cf. Rieck 1993: 35). However, attempts to produce systematic taxonomies have been rare. An exception are Rapoport and Guyer (1966) and Rapoport et al. (1976). They develop a taxonomy, which uses several solution concepts (dominant strategies, natural outcomes, Nash equilibria) and some factors that can make equilibria instable, such as threat-vulnerability. In this way they were able to rank matrix games according to the stability of their solutions.

Although the stability dimension is very important for theory, it is not of much relevance for applications in political science. Much more interesting for political science is a typology that allows different types of collective action problem to be distinguished. These are in fact the kind of distinctions that can be found in political science books (for example Schelling 1960: 88; Tay- 
lor 1987: 34-60; Tsebelis 1997: 61-68; Scharpf 1997: 69-79; Braun 1999). Prisoners' dilemmas, chicken, and assurance games are analysed with respect to the social problems of co-ordination, co-operation or inequality (Ullmann-Margalit 1977), and they are used to model empirical problems of the respective types.

In political science the most comprehensive attempt to classify 2 x 2 matrix games has been undertaken by Zürn (1992). Zürn distinguishes four types of games that represent what he calls "problematic situations": co-ordination games without distributional conflict, co-ordination games with distributional conflict, dilemma games, and rambo games. He uses three criteria for defining these types: the number of Nash equilibria, the Pareto-optimality of Nash equilibria, and the notion of a qualified Pareto-optimum, which serves to distinguish the rambo games (1992: 161-165). The classification presented here ends up with similar results, although on the basis of somewhat different criteria. In contrast to Zürn's taxonomy, it is a complete classification of all ordinal 2 x 2 games. However, the theoretically relevant types it yields are almost the same. Only two aspects of Zürn's taxonomy are contested: I argue that zero-sum games also represent problematic situations and that games without a Nash equilibrium are a class in their own right, with specific problems.

\subsection{A Classification of Strategic Form Games}

The classification of $2 \times 2$ matrix games developed here allows each game to be identified as belonging to a certain type of collective action problem. The aim of the classification is to find and distinguish all important types of collective actions problems relevant for political science insofar as they can be represented by strategic games. For this purpose it is necessary to classify all strategically distinct $2 \times 2$ matrix games according to certain properties, which reveal or represent collective action problems. The number of different matrix games is infinite, if cardinal payoffs are used. This is true, even if the games are restricted to two players and two strategies. Therefore it is impossible to tell how many individual games belong to one class of games. However, how can one be sure to include all relevant classes of games if there are an infinite number of games?

First of all, the ordinal formulation of payoffs is sufficient to capture differences in the strategic structure. Games with cardinal payoffs can be re-formulated into ordinal ones without any loss of information about the strategic structure. It is thus sufficient to classify all games with ordinal payoffs that are strategically different. In a 2 x 2 game with strict ordinal preferences there are four outcomes, which are ranked from 1 to 4 by the players. Each player can place the numbers into the four cells of the matrix in $4 !=24$ ways. Since there are two players, there are a total of $24 \times 24=576$ possible combinations of strict ordinal payoffs. Not all of them represent different strategic structures, however. The game matrices are strategically equivalent whenever only the rows, the columns, both rows and columns, or, in symmetric games, the players, are inter- 
changed. Taking this into account, only 78 strategically distinct games remain. ${ }^{2}$ If indifference about the outcomes or strategies is permitted, however, the number of strategically different games rises to 732 .

For the purpose pursued here it seems sufficient to work with the 78 strictly ordinal games and to treat them as representatives of 2 x 2 games in general. However, some interesting "borderline" games result if the players are allowed to be indifferent about the outcomes. Therefore I include some prominent games that are based on weak preferences. I have analysed all these games with respect to a number of game theoretic properties: symmetry or asymmetry, the number of Nash equilibria, the number of dominant strategies, the number of Pareto optimal outcomes, Paretooptimality at equilibria, conflict over outcomes, inequality of payoffs in Pareto-optimal outcomes and in equilibria, and the Kaldor criterion. ${ }^{3}$ The classification was developed on the basis of the results of this tentative analysis. Only four of these formal properties have proven to be crucial for a distinction between basic collective action problems.

These four properties of games and their equilibria are used firstly to classify matrix games and secondly to develop a typology of collective action problems. The properties can be easily identified for each game. The classification is empirically complete insofar as all 2 x 2 games can be subsumed under the disjunctive classes defined by the following four factors:

\section{1) Number of Nash equilibria in pure strategies (none, one, two or more)}

I have chosen to use the number of Nash equilibria in pure strategies as a criterion because mixed strategy equilibria seem empirically to be highly implausible solutions as long as one-shot games are considered. Pure strategies are what real actors will have in mind in the first place, and it is the number of pure strategy equilibria that determines whether problems of co-ordination or discoordination arise. If I had chosen to count the number of pure and mixed strategies, the distinct classes created would have remained the same anyway. The difference would be that the categories of this factor would have to be re-labelled as one mixed, one pure, three or more pure or mixed strategy equilibria.

\section{2) Pareto-optimality of the equilibria (optimal, sub-optimal)}

The Pareto-optimality and sub-optimality determines whether an outcome of the game entails conflict between individual and collective rationality. In cases of multiple pure strategy equilibria, it is distinguished whether all equilibria are Pareto-optimal or whether there are optimal and sub-optimal equilibria. This criterion cannot be applied to games that do not have an equilibrium in pure strategies.

2 The exact way of calculating this can be found in Rapoport, Guyer, and Gordon 1976: 14-17.

3 My work was greatly facilitated by the fact that these 78 games are provided by Rapoport, Guyer, and Gordon 1976: 23-30. 


\section{3) Conflict over the valuation of the outcomes (no, irrelevant, partial, pure)}

The conflict factor refers to the players' valuation of the four possible outcomes. No conflict means that the players rank all outcomes equally. Pure conflict means that the players' ranking of the outcomes is completely oppositional. In ordinal terms, the latter games are constant-sum games, in which the constant is 5 . Zero-sum games would be strategically equivalent in a cardinal game formulation. The group of games that can be classified as pure conflict games, however, is not restricted to constant- or zero-sum games, as the cardinal formulation allows for nonlinearity. ${ }^{4}$ Conflict over outcomes is irrelevant if both players rank the same outcome first, and different valuation occurs consequently only with respect to the second, third or fourth preference. For all other games, conflict is partial. Usually games in the last group are called "mixed motive" games.

\section{4) Equal payoffs in Pareto-optimal outcomes or equilibria (yes/yes, yes/no, no)}

The previous three factors constitute common ground in matrix game analysis. They have long served as criteria for the classification of games, and the strategic relevance of these attributes is obvious. The factor of inequality introduces a distributional dimension into the typology, which has thus far only been taken up in the Zürn classification. The factor is interesting from a political science perspective, since the distributional inequality of the outcome of a game may produce incentives to negotiate or to defect, even if the solution is rational (i.e., if it is an equilibrium). This has convincingly been shown by economic experimental research on fairness, beginning with "ultimatum bargaining" experiments. ${ }^{5}$ Including inequality goes beyond standard rational choice theory.

Equality of payoffs in the equilibrium is again measured ordinally. Ordinal comparisons of equality are problematic, however. The rejection of outcomes by players who judge them to be unfair is usually based on the inequality of cardinal payoffs. Outcomes, however, which have the same rank in the preference order of both players, may well be associated with very different cardinal payoffs, and vice-versa. If, in a battle of the sexes game, for example, the female gets her preferred equilibrium, it may well be that the male's overall cardinal utility is higher than the female's, as he values both spending an evening together and the female's preferred entertainment more than she herself does. On the other hand, it is not unreasonable to assume that players also judge an outcome as unfair where player 1 gets her first preference while player 2 gets only her third preference. This is implied in the usual interpretation of battle of the sexes games or other games with ordinal inequality in the equilibria. Implicitly we seem either to assume that there is the same cardinal utility associated with the same rank in the preference order, or we also evaluate distributional equality on the basis of ordinal comparison. Nida-Rümelin (1991), does so, for example, when he compares the outcomes of matrix games with ordinal payoffs in respect to justice. I will follow this tradition and work with the concept of ordinal equality in classifying

4 There may be games with four Pareto-optimal outcomes ranked completely oppositional and lying on a nonlinear curve.

$5 \quad$ Güth, Schmittberger, and Schwarze 1982; Fehr and Schmidt 1999. 
the 78 strategically different ordinal games. It should be clear, however, that the equality criterion makes more sense when applied to cardinal payoffs.

For the classification, I have used the equality criterion in the following way: there is equality if both players rank an outcome the same; there is inequality if they rank an outcome differently. Since I am concerned with what makes a game problematic, I am interested in the equality or inequality of outcomes that would be collectively desirable or that form an equilibrium. Thus, the inequality or equality of suboptimal outcomes or in outcomes which are no equilibria is of no relevance. If at least one player has a strong preference for equal solutions, a Pareto-optimal outcome may not be achieved, or an equilibrium may even be rejected by one of the players because it is characterised by inequality. Games are not problematic with respect to distributional justice if there is a Pareto-optimal equilibrium, which is associated with equal payoffs. They may become problematic, however, if the Pareto-optimal equilibrium is characterised by inequality, or if they have no equal Pareto-optimal outcomes at all - which implies that there are no equal Paretooptimal equilibria. Thus, two questions have to be asked:

1. Is there at least one Pareto-optimal outcome with equal ordinal payoffs (yes or no)?

2. If yes, is one of these outcomes a Nash equilibrium (yes or no)?

If the answer to the first question is no, there cannot be a Pareto-optimal and equal Nash equilibrium. The two questions are equivalent to applying the three criteria of Pareto-optimality, equality, and Nash equilibrium to a potential solution, respectively, to applying the two normative criteria Pareto-optimality and equality to the Nash equilibrium. The two questions create three groups of games:

those with Pareto-optimal and equal Nash equilibria (yes/yes), that is, both normative criteria are fulfilled in equilibrium;

those with equal Pareto-optimal outcomes, which are no equilibria ${ }^{6}$ or those with Paretooptimal equilibria, which are characterised by inequality ${ }^{7}$ or those which meet both conditions $^{8}$ (yes/no), that is, only one normative criterion is fulfilled in equilibrium;

those without any equal Pareto-optimal outcomes - and thus also without equal Paretooptimal Nash equilibria (no), that is, no normative criterion is fulfilled in equilibrium.

6 The only strictly ordinal example is the standard prisoner's dilemma.

7 The only strictly ordinal example a chicken game, which has a third and equal Pareto-optimal outcome (cf. table 2).

8 There are three strictly ordinal rambo games which meet both conditions (see example in table 2). 
Table 1: Classification of $2 \times 2$ Games and Typology of Collective Action Problems

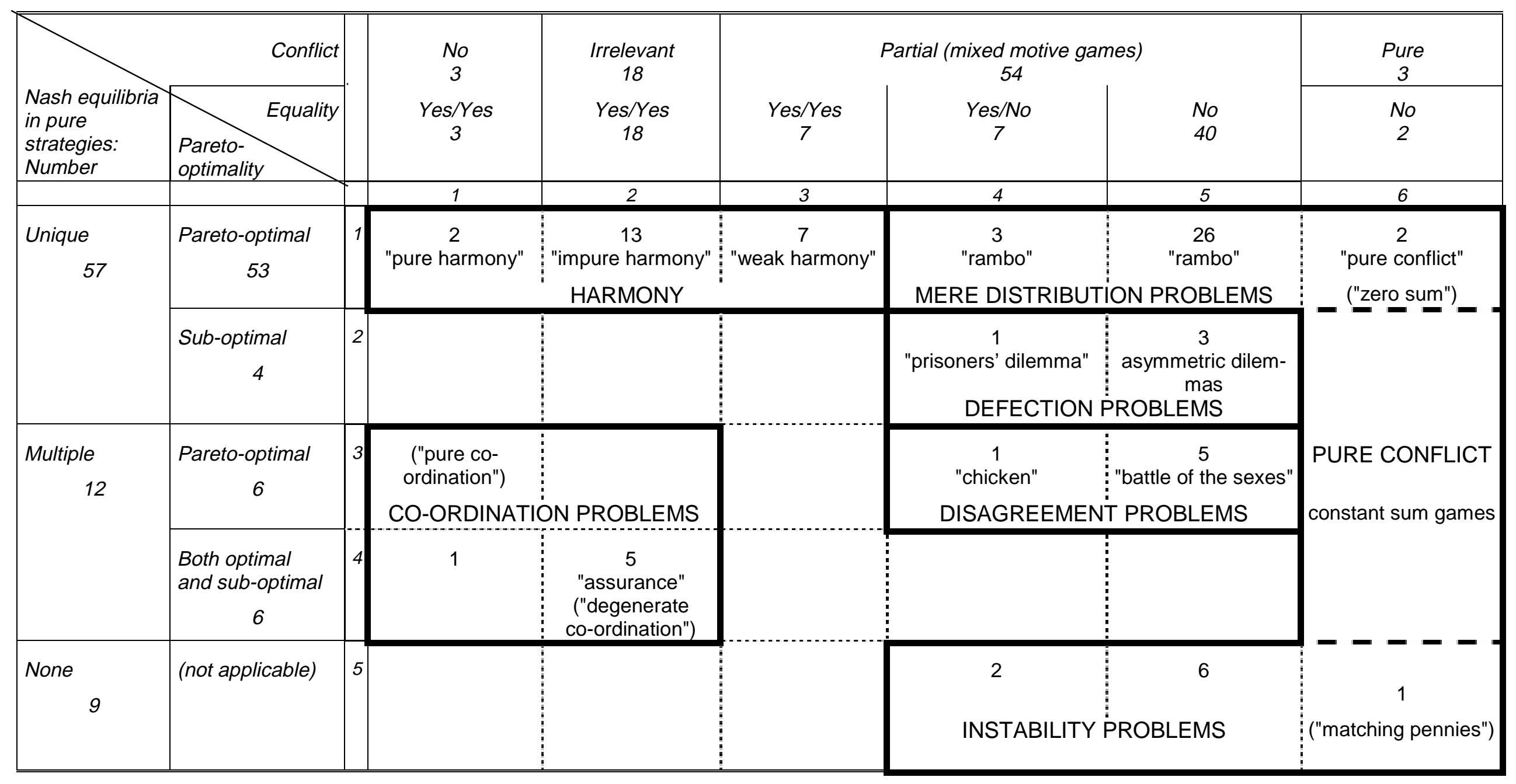


There is no distributional problem in the first group. The distributional problem ist most severe in the third group, as there are simply no optimal and equal solutions. In the second group, at least optimal and equal outcomes exist, which the players could try to achieve in a co-operative way.

The classification presented in table 1 is the product of these four factors. The complete product would include 72 cells. However, not all categories of each factor are applicable to all categories of all the other factors. For example, the two equilibria-related criteria cannot be applied to games without equilibrium in pure strategies. Other sub-classes are empirically empty; for example, in "no conflict" or "pure conflict" games equality does not vary. Thus, several rows and columns can be omitted and finally only 30 cells remain. In this way, the classification becomes more informative and remains at least as fine.

In table 1, factors (1) and (2) are given in the vertical dimension, and factors (3) and (4) in the horizontal dimension. The table shows first how the 78 strictly ordinal games are distributed over the 30 cells of the classification. The numbers given in the table refer to the number of strategically different strictly ordinal games in each class. Some prominent examples of each important class of games are mentioned in the cells in quotation marks. The matrices for most of them are given in table 2. Second, three additional games are given in brackets that are based on indifference to outcomes or strategies. These less well-known games are shown in table 3: the "pure coordination" game, the "degenerate co-ordination" game, and "matching pennies". Finally, a zero sum game in cardinal numbers is also classified and its matrix is given in table 3.

From this, it should become evident, that the classification is in fact a classification of all $2 \times 2$ matrix games, whether they are strictly ordinal, weakly ordinal or cardinal. However, only the 78 strictly ordinal ones, three weakly ordinal ones and one cardinal game has been actually classified here. In the following section, table 1 will be interpreted and a typology of the collective action problems involved will be derived. 
Table 2: Some Representatives of Important Classes of 2 x 2 Games

\begin{tabular}{cc|c|c|} 
& \multicolumn{2}{c}{ Player A } \\
& \multicolumn{1}{c}{1} & \multicolumn{1}{c}{2} \\
\cline { 3 - 4 } Player B & 1 & 1,1 & $2, \underline{2}$ \\
\cline { 3 - 4 } & 2 & $\underline{3}, 3$ & $\underline{4,4}$ \\
\cline { 3 - 4 } & & &
\end{tabular}

Pure Harmony

Player $A$

\begin{tabular}{cc|c|c|}
\multirow{2}{*}{ Player $B$} & 1 & \multicolumn{1}{c}{1} & \multicolumn{1}{c}{2} \\
\cline { 3 - 4 } & \multirow{2}{*}{2} & $2, \underline{4}$ & $\underline{3}, 3$ \\
\cline { 2 - 3 } & & $\underline{4,2}$ & 1,1 \\
\hline
\end{tabular}

Rambo

Player $A$

12

Player B

\begin{tabular}{|l|l|l|}
\cline { 2 - 3 } 1 & $\underline{4}, 3$ & $1, \underline{4}$ \\
\hline \multirow{2}{*}{3,1} & $\underline{2,2}$ \\
\hline
\end{tabular}

Asymmetric

Dilemma

\begin{tabular}{cccc|} 
& \multicolumn{2}{c}{ Player A } \\
& & \multicolumn{1}{c}{ Left } & \multicolumn{1}{c}{ Right } \\
\cline { 3 - 4 } Player B & Up & 1,1 & $\underline{4,2}$ \\
\cline { 3 - 4 } & & & \\
\cline { 3 - 4 } & Down & $\underline{2,4}$ & 3,3 \\
\cline { 3 - 4 } & & &
\end{tabular}

Chicken

\begin{tabular}{|c|c|c|c|}
\hline & \multicolumn{2}{|c|}{ Player A } \\
\hline & & Left & Right \\
\hline \multirow[t]{2}{*}{ Player B } & Up & $\underline{2,2}$ & 3,1 \\
\hline & Lovin & 1,3 & $\underline{4,4}$ \\
\hline
\end{tabular}

Assurance

Player $A$

\begin{tabular}{ll|c|c|}
\multicolumn{1}{c}{ Player $B$} & 1 & \multicolumn{1}{c}{1} & 2 \\
\cline { 3 - 4 } & & $\underline{2,3}$ & $\underline{4}, 1$ \\
\cline { 3 - 4 } & & $1, \underline{4}$ & 3,2 \\
\hline
\end{tabular}

Pure Conflict

Player $A$

\begin{tabular}{cc|c|c|}
\multicolumn{1}{c}{ Player $B \quad$ Up } & \multicolumn{1}{c}{ Left } & \multicolumn{1}{c}{ Right } \\
\cline { 3 - 4 } & \multirow{4}{*}{ Down } & 1,2 & $\underline{3,4}$ \\
\cline { 2 - 3 } & $\underline{4,3}$ & 2,1 \\
\hline
\end{tabular}

Battle of the

Sexes

\begin{tabular}{cc|c|c|} 
& \multicolumn{2}{c}{ Player A } \\
& \multicolumn{1}{c}{1} & 2 \\
\cline { 3 - 4 } Player B & 1 & $\underline{4}, 1$ & $1, \underline{2}$ \\
\cline { 3 - 4 } & 2 & $\underline{4}, \underline{4}$ & $\underline{2}, 3$ \\
\cline { 2 - 3 } & & &
\end{tabular}

Instability 
Table 3: Some 2 x 2 Games Involving Indifference and a Zero-Sum Game

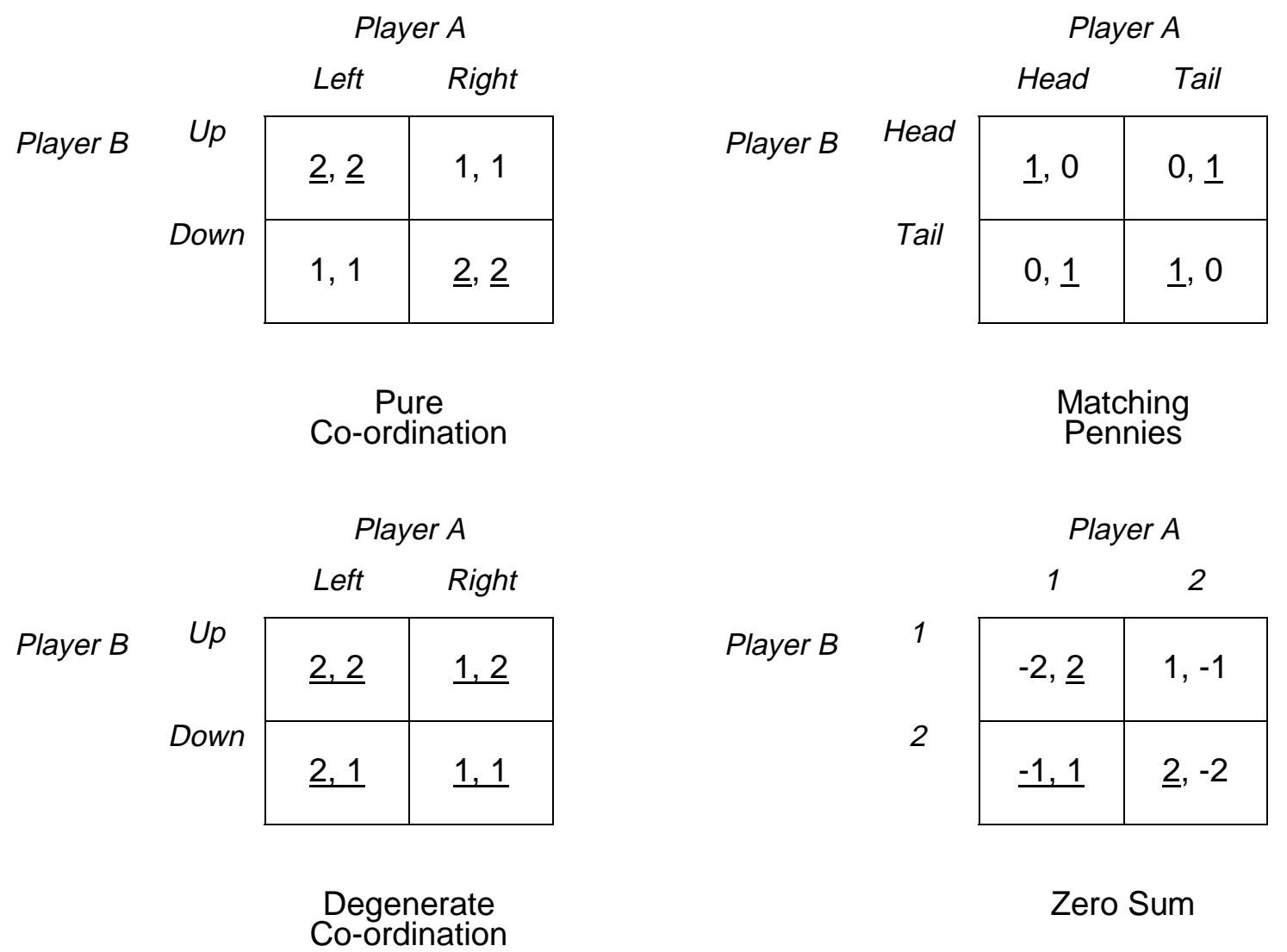

\subsection{Typology of Collective Action Problems}

The classification of matrix games makes it possible to distinguish between some basic types of collective action problem. Each type is defined by a certain combination of the four matrix game properties analysed. Although table 1 has 30 cells, there are only seven distinct classes of games which are interesting for collective actions problems. First, this is due to the fact that some of the cells are still logically or empirically empty (cf. table 1). Second, not all factors produce crucial distinctions in each of the sub-classes constituted by the other factors. Thus, several cells can be subsumed under one type of collective action problem. The types are marked in table 1 by bold frames. ${ }^{9}$ They have been labelled in accord with the dominant problem of a certain class of games. Sometimes classes of games combine several elementary aspects of collective action problems - for example a co-ordination plus a distribution problem - and thus form a new characteristic type. In the following, definitions and descriptions of the seven types are given. The

9 The frames mark only roughly the cells belonging to each type of collective action problem. They are not a correct and precise graphical representation of the types. In a correct representation, the types had to be given by the complete rows or columns, which represent one of the problematic aspects of collective action, or by intersections of those rows and columns. For example, all games in rows 3 and 4 are characterised by a coordination problem aspect, and all games in columns 4, 5, and 6 are characterised by a distributional problem. This includes the empty cells, as there may be weakly ordinal games which belong into these cells, but have not been classified here. However, in a black-and-white diagram this is difficult to viualize without creating optical confusion. 
number of strategically different games of the 78 ordinal games belonging to each type and each sub-type discussed, is given in brackets.

\section{1) Harmony}

The first type to be distinguished is the group of harmony games. They pose no collective action problem at all. The have a unique Nash equilibrium and thus a stable solution, which is Paretooptimal and maximises joint gains. There is no conflict concerning the valuation of outcomes (or conflict is irrelevant), and there is no inequality in the players' payoffs in the equilibrium. From a collective action perspective, the distinction between "pure" harmony games (2), where all outcomes are ranked equally by both players, and "impure" harmony games (13), where outcomes off the equilibrium path are ranked differently, is irrelevant. "Weak" harmony games (7) involve partial conflict and have two Pareto-optimal outcomes. However, these games are harmony games: that is, there is no inequality in the Pareto-optimal equilibrium.

\section{2) Mere Distribution Problems}

On the right hand side of the harmony games there is a group of games that have a unique and Pareto-optimal Nash equilibrium. They differ from harmony games only in that they possess a second Pareto-optimal outcome which is not an equilibrium, while the equilibrium is characterised by inequality. There is partial conflict over the valuation of outcomes. In the first group there are three games, in which there is inequality in the Pareto-optimal Nash equilibrium. In the second and larger group (26) each of the two Pareto-optimal outcomes (equilibrium or not) is characterised by inequality.

Both groups pose a distributional problem. It is merely distributional since, from the perspective of co-ordination, stability, and welfare effects, there are no problems with these games. They have been labelled "rambo" games by Zürn (1992: 209ff.). So far, they have not received much attention in the literature. There are two reasons why this presents a significant deficit in the political science literature: First, from an outside normative perspective, the inequality may be judged to be unfair. Second, from a positive perspective, if the strict game theoretic assumptions are given up, there may be an "irrational" incentive to defect, driven by the players' considerations of fairness, or an incentive to re-negotiate if communication is possible. Or, if both players accept a certain standard of fairness, some welfare might be traded off against some degree of fairness. Rambo games are probably very common and should not be neglected.

\section{3) Pure Conflict}

Further to the right in table 1 there are pure conflict games. In pure strategies they may have no equilibrium or just one equilibrium, and their equilibria (as all other outcomes) are by definition Pareto-optimal. Constant-sum games and zero-sum games are well-known examples. They pose solely distributional problems because their equilibria inevitably entail inequality between the 
players. In the cases of "no equilibrium" instability is a problem. Thus, these games belong to the mere distributional problems (2), and intersect partially with instability problems (1).

\section{4) Defection Problems (Dilemmas)}

The class of games below the rambo games entail a problem of defection (4). They have a unique Nash equilibrium. However, this is a sub-optimal equilibrium. There is partial conflict, and equilibria may result in either equality or inequality of players' payoffs. The collective action problem associated with this group of games is a conflict between individual and collective rationality. The Pareto-optimal outcome (which would be better than the equilibrium not only collectively but also individually) is not achieved by the players, as there is an individual incentive to deviate should the other player conform. The players are tempted to defect. The most well-known example is the prisoners' dilemma. Both the Pareto-optimal outcome and the Nash equilibrium are equality outcomes in the prisoners' dilemma.

However, there are also asymmetric dilemmas (3) in which the Pareto-optimal (non-equilibrium) outcome is characterised by inequality. The distributional problems that go along with these games make the situation worse: Even if the players could conclude a contract and enforce it, it would be difficult for them to agree to the Pareto-optimal outcome because that outcome is associated with inequality. Moreover, the Nash equilibria in these games may be equality outcomes. If the actors strongly value equality, they have one more reason to choose the sub-optimal equilibrium.

\section{5) Co-ordination Problems}

Below the harmony games there is a class of games that pose a co-ordination problem. The reason for this is the fact that these games have multiple equilibria in pure strategies. Some of them are no conflict games (1), and some are irrelevant conflict games (5). Both groups may have two Paretooptimal equilibria, as in the case of the "pure co-ordination" game. The "impure" co-ordination games have both an optimal and a sub-optimal equilibrium, such as the assurance game or the degenerate co-ordination game. There is no inequality in their equilibria, and they maximise joint gains in the optimal equilibria. The main problem for the players is to co-ordinate their strategies such that at least one of the equilibria, and hopefully the optimal equilibrium, will result.

\section{6) Disagreement Problems}

The next class, to the right of the co-ordination games and below the defection games, combines several problems. Games belonging to this class have two equilibria in pure strategies, and both are Pareto-optimal. However, both equilibria entail inequality - one disadvantages Player 1 and the other disadvantages Player 2. Thus players disagree about which equilibrium is to be selected. Examples are the battle of the sexes or the chicken game.

In its ordinal formulation the chicken game does not only have two Pareto-optimal Nash equilibria - both of which are inequality outcomes - it also has a third Pareto-optimal outcome, which 
is not an equilibrium, but which shows equality. ${ }^{10}$ The other five games in this class, including the battle of the sexes, possess only two Pareto-optimal outcomes. There is no problem with collective welfare in these games, but there is both a co-ordination and a distribution problem. Given this combination, the players have difficulties of finding an agreement. Both players prefer a different equilibrium, and there is thus a good chance that they will miss the equilibria. This is even true if both players act altruistically and pursue the other player's preferred equilibrium. If the players can communicate and thus eliminate the co-ordination problem, they will still have difficulties agreeing on one of the equilibria.

\section{7) Instability Problems}

The last group is formed by the so-called dis-coordination games (Rieck 1993: 53). These games have no pure strategy Nash equilibria. Therefore the criterion of Pareto-optimality cannot be applied. The main problem these games pose is instability. In these games, rational players generally want to prevent their strategies from intersecting - they dis-coordinate strategies. There is always a unilateral incentive to deviate from Pareto-optimal or joint gain-maximising outcomes. Most of these games are partial conflict games (8); however, they may appear in pure conflict situations as well (1). A classical example that allows for indifference is "matching pennies" (cf. table 3).

As mentioned above, matrix games have many more strategically relevant attributes: for example, symmetry or asymmetry; the Kaldor criterion (allowing evaluation of whether joint gains are maximised or optimised in equilibrium); the number of Pareto optimal outcomes; the number of dominant strategies; or the exact extent of conflict. In checking the sample of 78 games for whether they make any systematic and important difference and whether they create new and interesting sub-classes of games, these attributes have been included in the analysis. They make no such systematic difference. All these attributes may nevertheless influence the strategic structure of a game and the possibility of finding a stable solution or a solution that satisfies normative requirements such as welfare maximisation or distributional equality.

However, if too many dimensions are added to the classification, one ends up with a large number of classes, which are only subtly distinct when applied to collective action problems. Furthermore, one ends up with an immense number of logically or empirically empty classes, as some of the above mentioned criteria are only meaningful and strategically relevant if applied to certain sub-classes. These additional factors may be interesting in the analysis of a specific game. For example, when looking for a solution, it makes a slight difference whether a chicken or a battle of the sexes game is played, given the chicken game has a third Pareto-optimal outcome. The communalities between these games, however, are far more important. For the sake of parsimony, therefore, it is better not to include all these additional factors in the classification.

The additional criteria just mentioned have little regularity. Two examples shall be given. First, joint gains are maximised only in the Pareto-optimal equilibria of no conflict games and irrele- 
vant conflict games. For all other games, joint gains are never maximised, although there are sometimes equilibria that optimise joint gains. This is true, for example, for the equilibria of pure conflict games. Second, symmetry is only very weakly correlated with classes of games. All no conflict games (3) and all pure conflict games (3) are symmetric; a great number of irrelevant conflict games are symmetric (11 out of 18), while only a small number of partial conflict games are symmetric (17 out of 54). It is especially noteworthy that there is no correlation between asymmetric games and inequality in Nash equilibria.

The distribution of the 78 strictly ordinal preference games throughout the seven classes shows that the number of strategically different games in the various problematic classes is significantly higher than the number of games in the harmonic classes. There are altogether 22 pure, impure, and weak harmony games. There are three games that pose only moderate distributional problems, and 26 more severe rambo games, four defection games, six co-ordination games, six disagreement games, eight dis-coordination games, and three games of pure conflict. Thus, 56 of 78 strategically different games cause some sort of collective action problem. However, only four different dilemmas have been found. Thus, in the strictest sense of the term, there would be not many "collective action problems".

This result must be qualified, however. First, it is not clear what the distribution would look like if all 732 strategically different games were classified, that is, including the indifference games. Second, and more importantly, the empirical relevance need not at all be correlated with the theoretical distribution of strategically different game models throughout the types of collective action problem. As of yet, not much can be said about the empirical frequency of the collective action problems. It is true that representatives of most classes of collective action problems seem to have been frequently analysed and applied - with the exception of harmony, rambo, and instability games. The fact, however, that the prisoners' dilemma has received a great deal of attention in social science literature, while rambo games have not, does not make it possible to draw conclusions about the empirical relevance of the two games. This fact is probably a mere result of researchers perceptions and interests, and of the greater logical or normative attractiveness of the prisoners' dilemma. Moreover, systematic information on the empirical frequency of the different games is difficult to acquire.

The seven types of distinct $2 \times 2$ matrix games can be reduced to five types of collective action problems. First, harmony is a large class of games, but does not pose a collective action problem. Second, the pure conflict games pose either a mere distributional problem and can thus be subsumed under this type, or they pose an instability problem - which is in general combined with a distributional problem (cf. table 1). Thus, there are five types of collective action problem: coordination, distribution, defection, disagreement, and instability problems.

There is one objection that could be raised against the typology developed above: Are matrix games the right tool for representing collective action problems and are they a good basis for deriving solutions? 2 × 2 models cannot take into account many factors, which influence both the strategic constellations and the collective action problems. What happens if there are more than 
two actors and more than two strategies? What happens if there is perfect information instead of the imperfect information assumed in a matrix game? Finally, how does repeated interaction affect the strategic constellation? It is shown in the next section that most of these factors do not directly affect the basic strategic constellations and the types of collective action problem, but rather the potential for solving the collective action problems.

\section{The Resolution of Collective Action Problems}

\subsection{The Assumptions Underlying Matrix Games: A Good Basis for Deriving Solutions?}

As already mentioned, matrix game models may over-simplify reality. Often the empirical situation involves more than two players, who have more than two strategies at their disposal; the sequential structure of the game may be more complex, or games may be nested and have "variable payoffs" (Tsebelis 1997). Furthermore, the assumptions of complete, but imperfect information and the idea of a single-shot game may not be adequate for many situations. There will be instances where a single-shot, complete information $2 \times 2$ game is an adequate and sufficient model of the situation, while there will be other instances where one or several of the assumptions have to be altered. For predictions or for responses to these problems it is important to find out which of these "complications" change the strategic situation, making it easier or more difficult to solve a problem. Therefore, the most important restrictions of $2 \times 2$ games shall be discussed. ${ }^{11}$

\section{(1) Number of Strategies}

Matrix games are not restricted to two strategies. More than two strategies can be modelled, although it is impossible to model continuous strategies by matrices. A larger number of strategies or a different sequential structure of a game may or may not change the strategic structure, depending on the payoffs associated with the new strategies. More than two strategies lead to an increase in the number of outcomes and often also to an increase in the number of equilibria; however, this is not necessarily so. The children game "rock-paper-scissors" is a dis-coordination game with three strategies that have no equilibrium (cf. Kreps 1990: 11). It is very similar to the two-strategy game "matching pennies".

A larger number of strategies pose no general problem to the analysis undertaken here. As long as a situation can be modelled as a strategic form game, it will in principle display the same collective action problems, although they may be more complicated to verify. Thus, in an anarchic environment the presence of more than two strategies has no general effect on strategic constellations. In a co-operative setting, however, where players try to find a collective solution by communication or bargaining, a rise in the number of strategies may increase the potential of finding a solution. More strategies imply an increase in the number of alternative outcomes from

11 Most points made in this section are not new or original; they can be found in game theoretic literature and textbooks (cf. for example, Holler and Illing 1991; Gibbons 1992; Rieck 1993; Morrow 1994). 
which the players can select. Increasing the number of strategies is especially useful for solving distributional problems.

\section{(2) Number of Players}

Having a larger number of players does not generally change the strategic structure in a noncooperative game (Liebrand 1983; Chamberlin 1974). If many players play a symmetric game, that game retains the same strategic structure as the two-player game. This can be shown by using a standard prisoners' dilemma (cf. Sandler 1997: 31; Sandler 1998; Diekmann 1992). As long as we retain the assumption that the players are homogeneous, each individual player faces the same strategic structure, regardless of whether he or she plays "against" one or millions of players. This is also valid for other symmetric games. The same is true for asymmetric games if large groups of actors play the game in the two roles represented by the game. The individual upstream and downstream riparians of a river provide one example. The strategic structure is the same whether there are two or 200 riparians. The strategic structure may change, however, if additional players have different roles, for example, different strategies or different valuations of outcomes. This is because it introduces more heterogeneity into the game.

Taylor (1987: chapter 4) has shown that, in general, in infinitely repeated games the number of players does not change the strategic structure. In infinitely repeated prisoners' dilemma games both co-operation and defection are rational strategies and may appear. The number of players does not alter this result: Co-operation "could emerge in the supergame no matter how many players there are" [emphasis in original] (Taylor 1987: 104). However, since this is only valid under certain conditions, it is questionable whether these conditions are likely to be met empirically. The greater the number of players is, the greater the number of conditions that have to be satisfied. Thus, in infinitely repeated games, co-operation becomes less likely for large numbers of players. Moreover, it becomes much more difficult to monitor defections in large size groups with anonymous actors (Taylor 1987: 105; Liebrand 1983).

To sum up, the number of players may change the strategic constellation under certain conditions, although in other situations it may make no strategic difference. However, the number of players or strategies may affect the co-operative solutions to collective action problems. Sometimes it is easier to find solutions in a two player setting than in a context of millions of players; sometimes different solution mechanisms can be used in large groups. While two or three players may communicate to overcome a co-ordination problem, when there are large numbers of people, voting or a hierarchical rule may be more adequate. A disagreement problem can be solved by consensual bargaining between two or more players, but it may be more appropriately solved by voting or representation if hundreds of players are involved. With respect to the decisionmaking procedure, there is a big difference between two-player and three-players games: While the decision is necessarily a consensual one in a two-player game, majority voting is possible when three or more players are involved. This makes it possible to form coalitions. While numbers do not affect the basic strategic constellation in a non-cooperative environment, they do 
affect the strategic situation in a co-operative solution (Deutsch 1967; Riker 1962; Ordeshook 1992, Holler and Illing 1991).

The discussion of the effects of numbers on the strategic constellation and on the solution potential shows that some characteristics of a situation may be more important for solving a collective action problem than for the basic strategic constellation. This is especially true for two presumptions that have implicitly been made in the previous sections: imperfect information and singleshot interactions.

\section{(3) Imperfect Information}

Matrix games are simultaneous games. They presuppose that players cannot observe which strategy the other player will choose (imperfect information). This implies that there is no communication between the players. For most empirical situations this is not a realistic assumption. For example, the classical cover story of the battle of the sexes game seems highly artificial. In most cases, a couple that does not know how to spend the evening and does prefer different entertainments will be able to communicate. As a consequence the co-ordination aspect of the problem vanishes and a simple bargaining problem remains. In general, co-ordination problems tend to be easy to solve (or they do not arise at all), if communication between players is possible.

Similarly, if a game is played sequentially, and the second player is able to observe the first player's move, the co-ordination problem disappears. Sequential play may also imply a decision about other problematic aspects of the game. In an assurance game, if the first player chooses the sub-optimal equilibrium strategy, the second player does not have much choice but to follow. However, this is not a very likely result in this game. In a battle of the sexes game or a chicken game, the first player has a distributive advantage: He or she can choose his or her preferred equilibrium; the second player will rather follow than fall back on the sub-optimal noncoordination outcome.

Thus, if there is perfect information in a given situation, either because of the ability of the parties to communicate or because of sequential interaction, the strategic constellation differs from imperfect information. Communication and sequential play can solve the co-ordination aspect of problem; however, neither can solve problems of distribution or defection. Thus, sequential interaction and communication can be considered a solution mechanism for co-ordination problems.

\section{(4) Repeated Interaction}

So far only single-shot games have been considered. However, in many instances, repeated interaction changes the strategic structure and affects the potential for solution. Again, repetition might itself be considered a solution mechanism (Tsebelis 1997: 110). For infinitely repeated 
games folk theorems apply: A vast number of equilibria are possible. ${ }^{12}$ Any feasible average payoff across rounds can correspond to an equilibrium, provided it gives the players at least as much as they can guarantee for themselves without any co-ordination with other players. In an infinitely repeated prisoners' dilemma, for example, defection in each round of the game, as well as various co-operative strategies are equilibria. However, the folk theorem results are only valid when identical stage games are infinitely repeated by the same players.

The strategic structure does not change so much in finitely repeated games. For games with a unique Nash equilibrium in the stage game, like the prisoners' dilemma, the unique and subgame perfect Nash equilibrium of the repeated game is the equilibrium of the stage game. In the prisoners' dilemma mutual defection is the equilibrium. For games with multiple equilibria in the stage game - for example, the chicken game - finite repetition may lead to co-operation. This is because those Nash equilibria of the stage game that are least preferable for potential defectors work as a credible threat and can be used as a sanction against them (cf. Morrow 1994: 279; Holler and Illing 1991: 165). Thus, which consequences the finite repetition of games has depends on the strategic structure of the stage game.

The results of the theory of repeated games are based on the possibility of making credible threats to sanction a defecting player. Thus, repeated games imply repeated interactions between the same players. What is important is the long-lasting and non-anonymous relationship of the players: This allows them to threaten and sanction in the next stage and to identify the defectors in earlier rounds. Games that are played repeatedly against a large and anonymous population of other players and games where defections cannot be observed and attributed to a specific player do not satisfy the preconditions for a textbook repeated game, because sanctions are impossible, or they are at least highly implausible in the absence of a co-operative regime (Liebrand 1983; Frey and Bohnet 1996b; Coleman 1986).

On the other hand, sanctions are possible among small groups of players, for example, families, small communities, or EU member states, so long as they have an ongoing relationship and can detect deviations from implicitly or explicitly agreed upon co-operative strategies (Taylor 1987: 105). The logic of repetition applies here, even if the same players play different stage games.

There is another kind of repetition where the folk theorem logic will not work. Sometimes the same individual plays a game repeatedly, but he or she plays it against changing counterparts. These kinds of standardised situations appear often with co-ordination problems. If two people meet on a walkway too narrow for two, one of them has to step aside. A single individual experiences this situation often, but the pairs meeting consist of different persons each time. Individuals usually do not have the chance to sanction a non-cooperative bully by being non-cooperative themselves next time. Such a situation is not a repeated game in game theoretic terms. However,

12 There exist many folk theorems that are distinct in their exact range and formulation. Friedman (1971) was the first to formally prove a folk theorem. For a discussion of folk theorems and infinitely repeated games cf. Morrow 1994, chapter 9; Gibbons 1992, chapter 2; Taylor 1987, chapters 3 and 4. 
repetition enables the individuals to learn how to best behave in such a situation (Blume and Gneezy 1998).

The fact that games are often played repeatedly and that this may lead to many outcomes does not make the analysis of stage games superfluous. Not each game is infinitely repeated among the same and non-anonymous players. There are single-shot games, finitely repeated games, large and anonymous groups, and games in standardised situations. In all these cases the logic of folk theorems does not apply. There are also many cases where the same group of actors will only once be faced with a certain collective decision. Thus, the emphasis that some authors (for example Taylor 1987) put on supergames is not entirely justified. However, should a situation best be modelled as an infinitely repeated game, the theoretical task is to find institutions that help to select the collectively desirable equilibria from the many equilibria possible. In general, repetition will make it easier to solve collective action problems.

In finitely repeated prisoners' dilemmas in which players are uncertain about the other players' preferences, co-operation is a rational strategy (Kreps et al. 1982; Bates 1988). There is a folk theorem for incomplete information in finitely repeated games (Fudenberg and Maskin 1986): Any feasible and individually rational outcome can be achieved when there is only a small amount of uncertainty about the other player's payoff. This also includes co-operative strategies. This result is not driven by internal sanctioning and discounting but by uncertainty, since there is only finite repetition. Incomplete information can thus help to resolve dilemmas. It can be as effective as infinite repetition in making threats credible.

The discussion has shown that a change in strategic constellations may accompany changes in features described by the basic assumptions of $2 \times 2$ matrix games. However, the various types of collective action problems are still present. The situations exhibit the same problematic characteristics: inequality of outcomes, risk of co-ordination failure, defection incentives, problems of finding agreement, and instability. Furthermore, such changes in the matrix game features are often linked to the opportunities for finding solutions to the collective action problems. Some of them may even be interpreted as solution mechanisms themselves, for example, sequential play of a game or repeated interaction.

This leads to the substantive question important for the resolution of collective action problems: Is there a correlation between the types of collective action problem and particular solution mechanisms? Are certain collective action problems easier or more difficult to solve than others? Are there solutions that work successfully with one but not with another type of collective action problem? Which kinds of solution mechanisms exist, and which of them are relevant from a political science perspective? Is it necessary to develop specific political or institutional solutions for each type of collective action problem? In the remainder of the paper some preliminary considerations are presented concerning the relationship of the types of collective action problem and particular resolution mechanisms. They are intended to indicate the direction future research could take rather than to provide full answers to these questions. 


\subsection{Solution Mechanisms for Collective Action Problems}

There is a wide range of behavioral and institutional concepts for the solution of collective action problems. These solution mechanisms have been proposed by disciplines, as different as moral and political philosophy, sociology and social psychology, political theory, economics and game theory. In the following I will briefly discuss the most important solution proposals that have been made in these disciplines with a tentative view on their respective capacities to resolve or facilitate the resolution of different types of collective action problems.

Most of the philosophical and sociological solution proposals work at the individual level, and some of them are based on motivations. The classic philosophical solutions are based on altruistic behavior or on practical reason - the Shaftesbury and the Kantian solution, as they were called by Nida-Rümelin (1991). The classic sociological solution is based on norm-guided behavior: Individuals adhere to social norms concerning fairness and equity, as well as to pacta sunt servanda norms. The idea of internalized norms has been rejected by Coleman (1987). However there is sufficient evidence that individuals act according to norms and thus norms can play a role in the solution of collective action problems.

Many solutions proposed or implied by game theory and social scientists work also at the individual level. The game theoretic solutions include mixed strategies, correlated strategies, sequential play and repeated interaction. Mixed strategies are not really a solution to collective action problems; they are just another game equilibrium. In some games, however, mixed strategies can in fact offer a solution. Sequential play changes the structure of the games and may thus also offer a solution. Correlated strategies are a specific concept which makes it possible to find institutional solutions to co-ordination problems (Aumann 1974). Repeated interaction is rather a property of the situation. As we have seen, however, it very often opens up the possibility of finding a collectively desirable solution.

Two more mechanisms will be examined, which work at the individual level but are not game theoretic solutions: focal point solutions (Schelling 1960) and co-ordinative communication. They do not play a role in non-cooperative matrix game theory, since there is no communication in matrix games, and focal point solutions are difficult to incorporate into the theory. Like the game theoretic mechanisms, they are based on rational expectation rather than on other motivations. They may help, whenever individuals have an incentive to co-ordinate their behaviour. They do not require that individuals conform to norms, or that there is any kind of coercion or enforceable contract involved.

Most other solution proposals belong to the realm of politics: They are procedural or institutional solutions and are based on collective decision-making and coercion. The classic political theory proposal is the Hobbesian solution (Nida-Rümelin 1991). It includes the delegation of all powers to a sovereign who can then coerce the individual actors into co-operative behavior. Similarly, game theory has stressed the necessity of external power, hierarchically imposed rules, and enforceable contracts in case of collective dilemmas. The hierarchical solution emphasizes the en- 
forcement of a given co-operative solution. It overlooks, however, that in many collective action problems, especially those which have a distributional element, it is not a priori clear which outcome is collectively desirable. Agreeing on a collective goal is problematic in the first place.

The Hobbesian solution is thus not fully equivalent with the political solution of the problem. If one assumes that a dictator will enforce the solution (which would be the true Hobbesian solution), there is no problem in finding agreement, although there is no certainty that the dictator will really enforce the outcome that maximises collective welfare. If one assumes a collective that is willing to delegate power to a central institution that can then enforce a collective decision (which is a standard political solution), the first step is still problematic: that is, there is a problem to find an agreement how to solve the problem. Therefore political solutions encompass two groups of mechanisms: mechanisms of finding agreements, like bargaining or voting, and mechanisms of enforcing agreements, like monitoring and sanctioning defections.

Four groups of mechanisms are distinguished in table 4: individual solutions based on the motivations of actors, individual solutions based on rational expectations, collective solutions, and coercive solutions. How do these mechanisms relate to the five basic types of collective action problem as developed above? Are all mechanisms capable of solving all or at least some types of problem? Are there in fact mechanisms which can solve all types of problem? Can all mechanisms solve at least some types fully or can they facilitate the solution? Are there mechanisms which are especially well suited to solve certain types of problem? A brief discussion follows of what the virtues of various kinds of mechanisms are. All solution have in common that the change the strategic structure of the game and thus the original problem.

Table 4: List of Solution Mechanisms

\begin{tabular}{l|l}
\hline Group of Mechanism & Solution Mechanisms \\
\hline Individual solutions & \\
- based on motivations & Altruism \\
& Equity and fairness norms \\
& Pacta sunt servanda norms \\
\hline Individual solutions & Mixed strategies \\
- based on rational expectations & Focal points \\
& Social conventions \\
& Correlated strategies \\
& Sequential play \\
\hline Collective solutions & Infinite and finite repetition \\
- decision-making mechanisms & Co-ordinative communication \\
& Bargaining \\
\hline Coercive solutions & Voting \\
- enforcement mechanisms & Enforceable contracts \\
& Hierarchically set and enforced rules \\
& Monitoring \\
\hline \hline
\end{tabular}


The motivational solutions can obviously facilitate two problematic aspects, namely distribution and defection problems. If fairness norms and pacta sunt servanda norms are present the problems of defection and distributional conflict will be less severe. However, these mechanisms cannot be relied upon to completely resolve distributional and defection problems, as in general not everyone will adhere to the norms, at least not in every situation. When distribution and defection aspects form only part of the problem, as in disagreement and instability problems, the motivational mechanisms are not sufficient to solve the collective action problem. Neither altruism nor social norms can resolve co-ordination problems or facilitate their resolution. Altruism does not help to overcome a battle of the sexes problem: the partners may miss each other, even if they go to the other's preferred entertainment.

Most individual strategies based on rational expectations work with co-ordination problems, while only some work with the other types of problems. This is true for mixed strategies, correlated strategies (Aumann 1974; Tsebelis 1997: 69; Arce and Sandler 2001), sequential interaction, focal points and social conventions (Schelling 1960: 89-118; Young 1998). All these mechanisms can co-ordinate actions such that the suboptimal outcome of "missing each other" is avoided. Playing mixed strategies can solve co-ordination, disagreement and even instability games. Mixed strategies are a practicable solution only when there is repeated interaction. In single-shot games they will only lead to normatively desirable outcomes by co-incidence. The other mechanisms of this group can also contribute to the solution of disagreement problems insofar as they overcome the co-ordination aspect. In most cases, however, the distributional aspect remains to be solved by additional mechanisms.

Finite repetition can produce stable, optimal and equal outcomes in co-ordination and disagreement games. However, other strategies and outcomes are also possible. Infinitely repeated interaction is the only non-cooperative mechanism that can theoretically solve all types of problems. However, it is not a sufficient solution to all problems, because co-operative strategies will not necessarily arise in infinitely repeated games. Defection is always possible, as well. Whether cooperation arises depends on theoretical and empirical conditions (Taylor 1987). However, the resolution of all types of problems will be facilitated if there is repeated interaction, since the potential of internal sanctioning opened up by repetition furthers co-operation.

Collective and coercive mechanisms can solve all types of problems, although they are not always necessary tools. In co-ordination problems they imply "overkill". However, in case of great numbers of players the hierarchical regulation of co-ordination may be efficient. Traffic rules provide an example. Pure communication can solve co-ordination problems, but not the other types of problem, although it may help to solve them (Crawford 1990). Bargaining and voting can solve disagreement games so that there are no incentives to defect after the agreement. However, bargaining and voting are not sufficient when there are strong incentives to defect from a bargained (or otherwise decided upon) agreement. Self-enforcing bargained solutions are thus possible, but may not be self-enforcing in all cases. Additional coercive mechanisms are needed. 
In any case, in order to solve most distributional problems, and especially defection and instability games, bargaining and voting must be combined with enforceable contracts. This is equivalent to the presence of an enforcement body that has the necessary powers. It can be assumed that hierarchically set rules (laws) and enforceable contracts are backed up with those powers. Monitoring and sanctioning within a collective can perform the same function (Ostrom 1990: 94100). Monitoring alone is not sufficient in general, although it may work in small groups combined with repeated interaction or the presence of norms. In all other cases monitoring is rather a precondition for sanctioning. The threat of a sanction and the power to sanction must be present.

In conclusion, if the five types of collective action problem are looked at from the perspective of solution mechanisms, they fall into two main categories:

(1) Problems of co-ordination: They can be resolved by a variety of non-cooperative mechanisms that work at the individual level and are based on rational expectations. It is, however, also possible to solve them by political mechanisms. When dealing with large groups this may be more efficient.

(2) Problems involving conflict: These problems, including disagreement, distribution, defection, and instability, can best be solved by collective and coercive mechanisms. In disagreement problems it will sometimes be possible for the collective to find self-enforcing agreements. However, in the other three types of collective action problem, coercion is necessary. The presence of altruism or social norms may facilitate the resolution of the problems. Since it cannot be relied upon to be effective, it will usually complement the political mechanisms. However, it can replace them under very specific circumstances, for example, in small groups with high degrees of altruism or norm-guided action.

These preliminary considerations show that not all solution mechanisms proposed by the various disciplines concerned with collective action problems are equally effective with respect to the different types of collective action problem. However, more detailed research is necessary to show which role the individual mechanisms can play in the resolution of the five types of collective action problem. Both theoretical deduction and empirical testing can help to find out which problem can best be tackled with which mechanism.

\section{Summary}

A typology of collective action problems was developed on the basis of a classification of $2 \times 2$ matrix games according to four formal properties of the games: the number of Nash equilibria, the optimality of the equilibria, the extent of conflict, and the equality of outcomes. Five types of collective action problem were distinguished, which represent different combinations of four problematic aspects in collective action: problems of efficiency, of co-ordination, of distribution and of stability: 
- Mere distribution problems imply efficient and stable outcomes. Their only problem is that their Pareto-optimal outcomes result in inequality, which may lead to manifest conflict.

- Pure and impure co-ordination games entail the problem that the actors may not be able to co-ordinate their strategies at the desirable equilibrium. Some of these games, such as assurance games, also have an efficiency aspect, since they have optimal and sub-optimal equilibria. There is a risk that the optimal equilibrium will not be achieved.

- Defection problems have a sub-optimal equilibrium, in some cases combined with inequality in the Pareto-optimal outcome. Their most unpleasant feature is that even if the parties agree to play the co-operative strategies, they have an incentive to defect afterwards. Defection problems may also reveal a secondary distributional problem.

- Disagreement problems combine both the co-ordination and distribution aspect. They have several equilibria, each preferred by other actors. Even if the co-ordination aspect can be overcome by communication or other means, the parties find it difficult to attain agreement.

- Instability problems are posed by games that have no equilibrium in pure strategies. There will be no stable solution to these problems, because the actors try to dis-coordinate their strategies. Instability problems usually combine the instability and the distributional aspect.

Second, a number of variables were examined which cannot be analysed within the framework of $2 \times 2$ matrix games. Changes in the basic assumptions of $2 \times 2$ matrix games may lead to changes in strategic constellations. However, the basic types of collective action problems will still be present in games which cannot be modelled as $2 \times 2$ games. Furthermore, such changes are often linked to the opportunities for finding solutions to the collective action problems. Additional strategies may change the structure of the non-cooperative game, but they may also facilitate a co-operative solution. The presence of more than two players does not in general change the strategic constellation - neither in one-shot nor in repeated games - but it may change it in particular instances. Large numbers of players will, however, often make it more difficult to resolve collective actions problems. The strategic structure is different if there is perfect information in a game. Perfect information especially facilitates the solution of co-ordination problems. Interaction between the same players in infinitely repeated games in general facilitates the resolution of collective action problems. However, it does not guarantee co-operation, especially if there are a large number of anonymous players. The differences between types of collective action problem present in the stage games disappear in infinitely repeated interactions. However, these differences still exist in finitely repeated games or in standardised repeated situations.

Third, various solution mechanisms were discussed which can resolve the types of collective action problem. Not all mechanisms can solve all types of collective action problem. Some mechanisms are appropriate for solving particular types of problem: For example, focal points, mixed strategies, social conventions, communication and sequential interactions can resolve coordination problems. The same mechanisms are no help, however, for distribution and defection problems. On the other hand, mechanisms that change motivational and behavioural assumptions 
(altruism and fairness norms) can contribute to the solution of disagreement, distribution and defection games. However, these mechanisms cannot be relied upon to fully resolve the problems. Repeated interaction also provides the actors with opportunities to resolve the collective action problems, because in infinite repetition co-operation is a rational strategy. Since defection is also rational in most cases, repetition may help; however, it will not necessarily resolve all collective action problems. Thus, for most types of collective action problem we are left with the political solution mechanisms: bargaining or voting as mechanisms for finding agreement in a collective; the hierarchical setting of rules and the enforcement of contracts, relying on monitoring and sanctioning, as mechanisms for enforcing the agreements. The presence of repeated interaction, as well as the presence of altruism and social norms can help to solve these problems. These mechanisms are important, as in many situations - for instance in international politics political and especially coercive solutions are not feasible or not desirable because they cause also high costs. 


\section{References}

Arce, M. Daniel G. and Sandler, Todd (2001). "Transnational Public Goods: Strategies and Institutions." European Journal of Political Economy 17: 493-516.

Aumann, Robert (1974). "Subjectivity and Correlation in Randomized Experiments." Journal of Mathematical Economics 1: 67-96.

Bates, Robert (1988). "Contra Contractrarianism: Some Reflections on the New Institutionalism." Politics \& Society 16: 387-401.

Braun, Dietmar (1999). Theorien rationalen Handelns in der Politikwissenschaft. Opladen: Leske + Budrich.

Blume, Andreas and Gneezy, Uri (1998). An Experimental Investigation of Optimal Learning in Coordination Games. WZB discussion paper FS IV 98-12. Berlin: Wisenschaftszentrum für Sozialforschung.

Coleman, James S. (1986). "Social Structure and the Emergence of Norms Among Rational Actors." In Paradoxical Effects of Social Behaviour. Essays in Honor of Anatol Rapoport (Andreas Diekmann and Peter Mitter, eds.), pp. 55-83. Heidelberg-Wien: Physika.

Coleman, James S. (1987). "Norms as Social Capital." In Economic Imperialism. The Economic Approach Applied Outside the Field of Economics (Gerard Radnitzky and Peter Bernholz, eds.), pp. 133-55. New York: Paragon House Publishers.

Crawford, Vincent P. (1990). "Explicit Communication and Bargaining Outcomes." American Economic Review 80: 213-19.

Deutsch, Morton, Epstein, Yakov, Canavan, Donnah and Gumpert Peter (1967). "Strategies of Inducing Cooperation - An Experimental Study." Journal of Conflict Resolution 11: 34560.

Diekmann, Andreas (1992). "Soziale Dilemmata. Modelle, Typisierungen und empirische Resultate." In Theorie, Daten, Methoden: neuere Modelle und Verfahren in den Sozialwissenschaften (H.-J. Andress, ed), pp. 176-203. Rieden: WB-Druck.

Elster, Jon (1985). "Rationality, Morality, and Collective Action." Ethics 96: 136-55.

Fehr, Ernst and Klaus M. Schmidt (1999). "A Theory of Fairness, Competition, and Cooperation." Quarterly Journal of Economics 114: 817-68.

Frey, Bruno S. and Bohnet, Iris (1996). Tragik der Allmende. Einsicht, Perversion und Überwindung." Kölner Zeitschrift für Soziologie und Sozialpsychologie, Special Issue 36, 292 307. 
Friedman, Jeffrey (1971). "A Noncooperative Equilibrium for Supergames." Review of Economic Studies 38: 1-12.

Fudenberg, Drew and Maskin, Eric (1986). "The Folk Theorem in Repeated Games with Discounting or with Incomplete Information." Econometrica 54: 533-54.

Genschel, Philipp and Thomas Plümper (1996). "Kommunikation und Kooperation in der internationalen Bankenregulierung." Zeitschrift für Internationale Beziehungen 3: 225-53.

Gibbons, Robert (1992). Game Theory for Applied Economists. Princeton, N.J.: Princeton University Press.

Güth, Werner, Rolf Schmittberger, and Bernd Schwarze (1982). "An Experimental Analysis of Ultimatum Bargaining" Journal of Economic Behavior and Organization 3: 367-88.

Holler, Manfred (1983). "Collective Action, Rational Man and Economic Reasoning." Quality and Quantity 17: 163-177.

Holler, Manfred J. and Gerd Illing (1991). Einführung in die Spieltheorie. Berlin et al: Springer.

Kreps, David M, Paul Milgrom, John Roberts, and Robert Wilson (1982). "Rational Cooperation in the Finitely Repeated Prisoners' Dilemma." Journal of Economic Theory 27: 245-53.

Kreps, David M. (1990). Game Theory and Economic Modeling. Oxford: Clarendon Press.

Liebrand, Wim B. G. (1983). "A Classification of Social Dilemma Games." Simulation \& Games 14: 123-38.

Liebrand, Wim B. G: (1996) and Messick, David M. Frontiers in Social Dilemmas Research. New York: Springer.

Morrow, James D. (1994). Game Theory for Political Scientists. Princeton, N.J.: Princeton University Press.

Nash, John (1951). "Non-Cooperative Games." Annals of Mathematics 286-295.

Neumann, John von and Oskar Morgenstern (1943). Theory of Games and Economic Behavior. Princeton, N.J.: Princeton University Press.

Nida-Rümelin, Julian (1991). "Practical Reason or Metapreferences? An Undogmatic Defense of Kantian Morality." Theory and Decision 30: 133-62.

Ochs, Jack (1995). "Coordination Problems" In The Handbook of Experimental Economics (John H. Kagel and Alvin E. Roth, eds), pp. 111-94. Princeton, NJ: Princeton University Press. 
Olson, Mancur (1965). The Logic of Collective Action: Public Goods and the Theory of Groups. Cambridge, MA: Harvard University Press.

Ordeshook, Peter C. (1992). A Political Theory Primer. New York-London: Routledge.

Ostrom, Elinor (1990). Governing the commons. The evolution of institutions for collective action. New York: Cambridge University Press.

Rapoport, Anatol (1988). "Experiments with N-Person Social Traps: I. Prisoner's dilemma, weak prisoner's dilemma, volunteer's dilemma and largest number." Journal of Conflict Resolution 32: 457-72.

Rapoport, Anatol and Melvin Guyer (1966). "A Taxonomy of 2 x 2 Games." In General Systems. Yearbook of the Society for General Systems Research XI: 203-14.

Rapoport, Anatol, Melvin J. Guyer, and David G. Gordon (1976). The 2 x 2 Game. Ann Arbor: University of Michigan Press.

Rieck, Christian (1993). Spieltheorie. Einführung für Wirtschafts- und Sozialwissenschaftler. Wiesbaden: Gabler.

Riker, William H. (1962). The Theory of Political Coalitions. New Haven, Conn: Yale University Press.

Runge, Ford C. (1984). "Institutions and the Free Rider: The Assurance Problem in Collective Action." The Journal of Politics 46: 154-81.

Scharpf, Fritz W. (1997). Games Real Actors Play. Actor-Centered Institutionalism in PolicyResearch. Boulder: Westview Press.

Schelling, Thomas (1960). The Strategy of Conflict. Cambridge, MA: Harvard University Press.

Taylor, Michael (1987). The Possibility of Cooperation. Cambridge-New York-Paris: Cambridge University Press.

Tsebelis, George (1997). Nested Games. Rational Choice in Comparative Politics. Berkely: University of California Press.

Ullmann-Margalit, Edna (1977). The Emergence of Norms. Oxford: Clarendon Press.

Zürn, Michael (1992). Interesssen und Institutionen in der internationalen Politik. Grundlegung und Anwendungen des situationsstrukturellen Ansatzes. Opladen: Leske+Budrich. 